\title{
Syphilitic retinitis in a homosexual man with concurrent HIV infection: case report
}

\author{
P W JOYCE,* K R HAYE, $†$ M E ELLIS $\ddagger$ \\ From the *Manchester Royal Eye Hospital, the †Department of Genitourinary Medicine, Manchester Royal \\ Infirmary, and the $\ddagger$ Regional Department of Infectious Diseases and Tropical Medicine, Monsall Hospital, \\ Manchester
}

SUMMARY A case is reported of secondary syphilis with ocular signs in a patient who had antibodies to human immunodeficiency virus (HIV) and hepatitis B infections and from whose urine cytomegalovirus was cultivated. Treatment with penicillin resulted in rapid recovery from his retinitis and uveitis. The case highlights the importance of testing for syphilis in all patients with HIV infection.

With the rising incidence of AIDS throughout the world, other sexually transmitted diseases (STD) tend to be overlooked outside clinics of genitourinary medicine (GUM) and departments of infectious diseases. As syphilis has a similar mode of transmission, however, it is not unlikely for it to appear in the presence of human immune deficiency virus (HIV), especially in people at high risk of acquiring STD. Syphilitic lesions in the eyes of homosexual patients with AIDS have been reported from America. ${ }^{12}$ We report on a patient who had secondary syphilis with ocular signs in addition to having antibody to HIV.

\section{Case report}

A homosexual man aged 24 presented to the casualty department at the Manchester Royal Eye Hospital in May 1987 with soreness of the right eye of two weeks' duration. He also complained of mild photophobia. His general health was good, except that he had noticed some hair loss from his scalp in the last two or three months. He was not receiving any medication.

On examination his visual acuity was $6 / 9$ and $6 / 6$. Bilateral perilimbal injection with flare and inflammatory cells $(3+)$ was seen in both anterior chambers. Keratic precipitates were also present in the right eye. Bilateral vitritis, which was worse on the right, was also seen. Fundoscopy of the right eye showed retinitis in the inferonasal quadrant with

Address for reprints: Mr P W Joyce, Department of Ophthalmology, University of Manchester, Lister House, Nelson Street, Manchester M13 9WH

Accepted for publication 30 May 1989 diffuse retinal oedema and several small round yellowish-grey exudates. Evidence of localised vasculitis (fig 1) was also present. The optic disc appeared to have blurred margins, though the vitreous haze precluded a precise view (fig 2 ). The left retina was unaffected.

Syphilis was considered in the differential diagnosis, and we immediately arranged for him to have a chest $x$ ray and took blood for a full blood count, the erythrocyte sedimentation rate, and standard serological tests for treponemal infections (the Venereal Disease Research Laboratory (VDRL) and fluorescent treponemal antibody absorption (FTAABS) tests and the Treponema pallidum haemagglutination assay (TPHA)). While awaiting the results of these investigations, topical ocular treatment was commenced immediately with dexamethazone $1 \%$ every two hours, cyclopentolate $1 \%$ every six hours, and betamethazone ointment at night to both eyes.

The patient was reviewed three days later when the results of his tests became available; they were positive in the FTA-ABS test and the TPHA and in the VDRL test at a titre of more than $1 / 256$. His erythrocyte sedimentation rate was $36 \mathrm{~mm}$ in the first hour. His full blood count and differential were within normal limits. His chest $x$ ray also showed no abnormality. His visual acuity had deteriorated to $6 / 24$ and 6/12 respectively. The anterior uveitis was worse in both eyes, and the view of the right fundus was further obscured by the vitritis. In his left eye the vitritis appeared to be unchanged, and the retina was still unaffected. The patient was immediately referred to the genitourinary medicine (GUM) clinic.

On examination in the GUM clinic he stated that he was a practising homosexual and had had several 


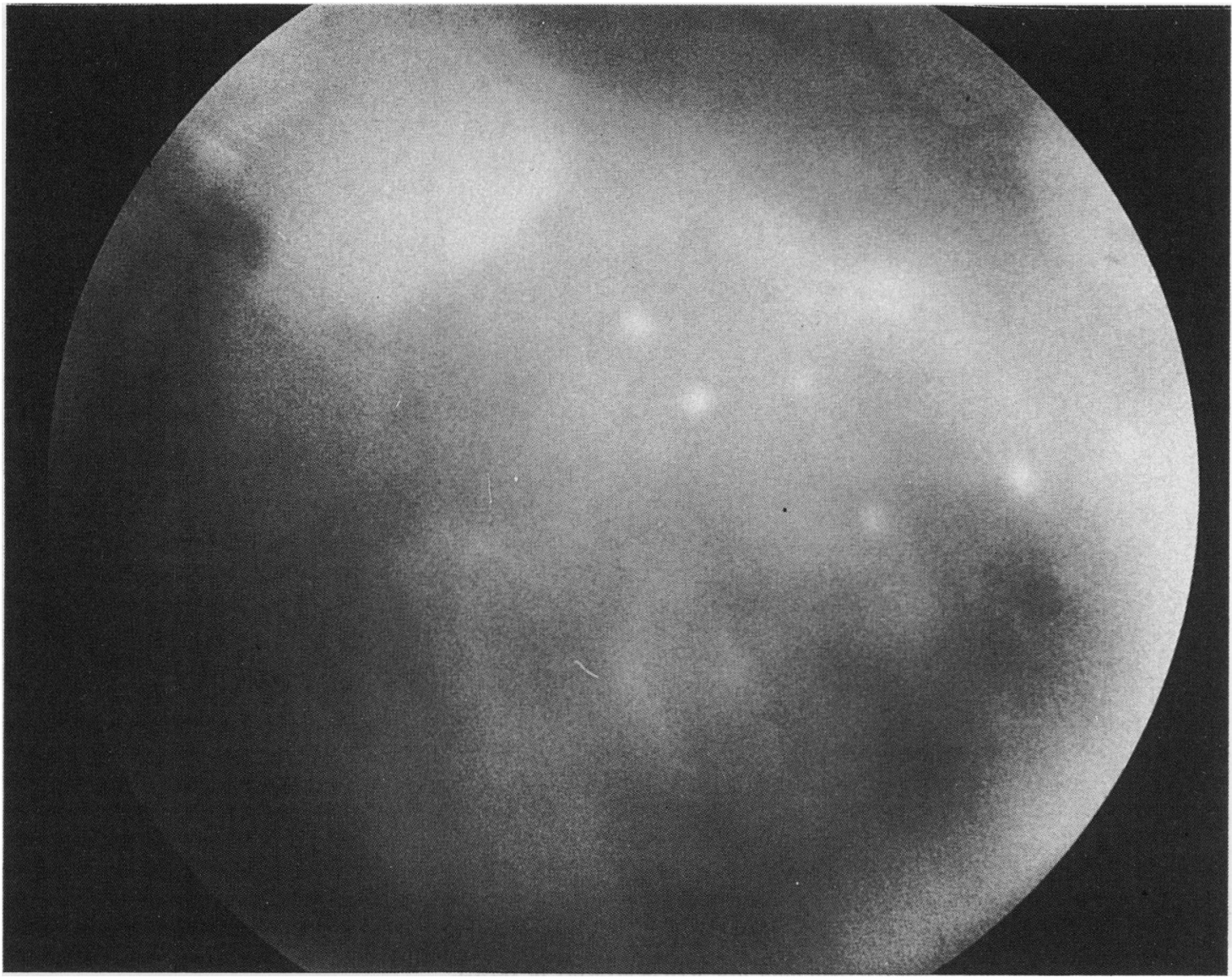

Fig 1 Fundus photograph of the right inferonasal quadrant showing evidence of retinal oedema, several round exudates, and retinal vasculitis (in upper part of photograph).

casual male consorts in the previous two years. Most of the time he was a passive (anorectal receptive) partner. For the previous six months he had had a regular sexual partner who had been treated for primary syphilis in March 1987 and had been found to have antibodies to HIV in February 1987. Our patient was therefore counselled and tested for antibodies to HIV, hepatitis B surface antigen, cytomegalovirus (CMV), and toxoplasmin. His serological tests for syphilis were repeated.

Examination showed a thin man who had discrete non-tender inguinal and axillary lymphadenopathy. He had perianal condylomata lata and alopecia affecting the scalp, eyebrows, and limbs. Except for tinea versicolour on the trunk, he had no other mucocutaneous lesions. His liver and spleen were not palpable, and the rest of the physical examination, apart from the visual findings, showed no abnormality.
Secondary syphilis was diagnosed, and treatment was started with intramuscular injections of penicillin ( $0.9 \mathrm{MU}$ a day and $2 \mathrm{G}$ oral probenicid a day for a total of three weeks). To protect him from a JarischHerxheimer reaction, however, he was given oral prednisolone $\mathbf{4 0 ~} \mathrm{mg}$ a day for three days before his penicillin injections. The prednisolone was then gradually tailed off by $5 \mathrm{mg}$ a day, and eventually stopped after eight days. During the course of his treatment he had severe oral candidiasis, for which he received nystatin pastilles (one three times a day for three weeks) with little benefit.

The results of his investigations were as follows: the titres in the VDRL and FTA-ABS tests and the TPHA remained unchanged; he had antibody to HIV; he had hepatitis B surface antigen, but no antibody core IgM; he had antibody to CMV but not IgM. CMV was also detected in the urine. The titre of antibody to toxo- 


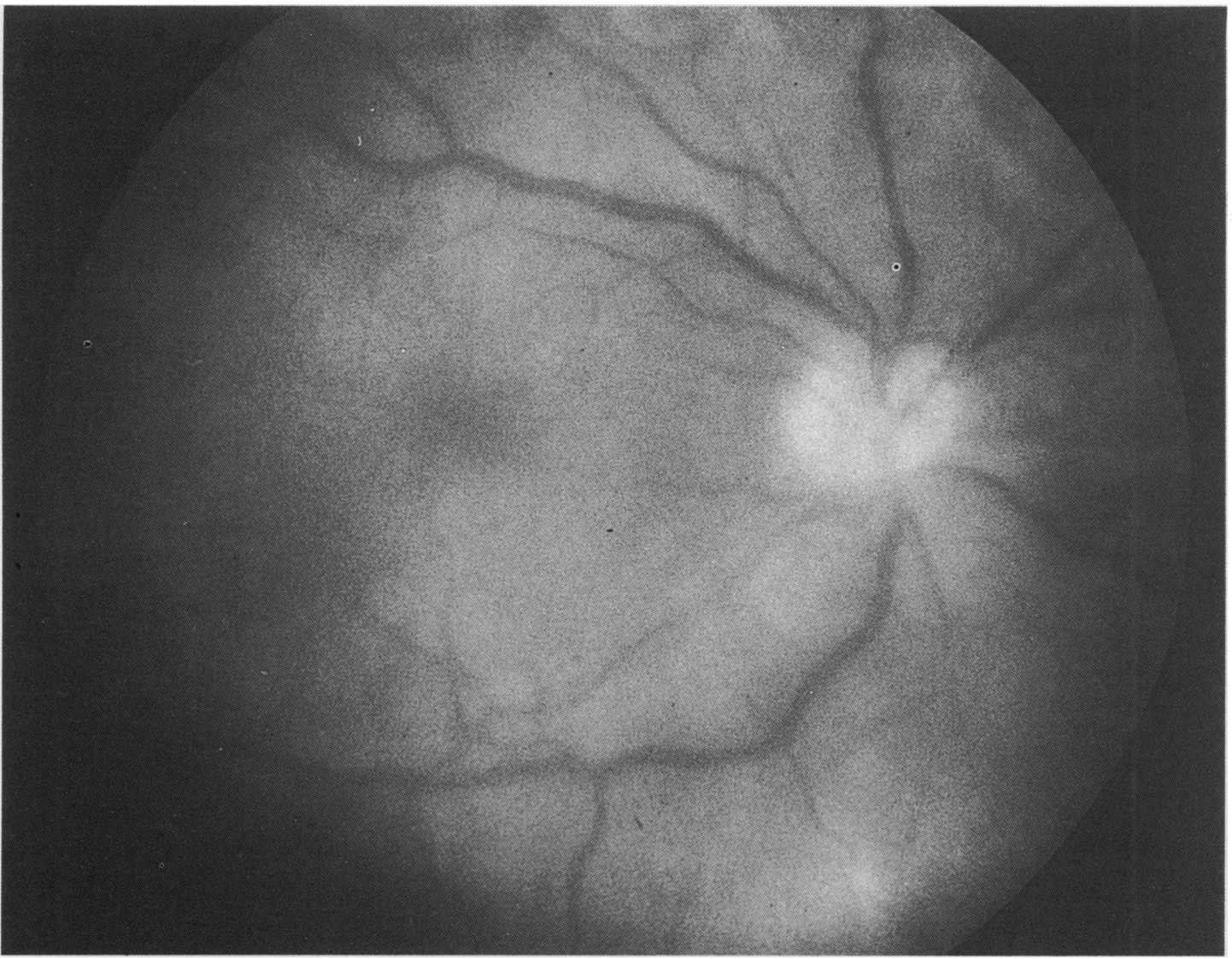

Fig 2 Fundus photograph of blurred right optic disc seen in presence of vitritis.

plasmin was not significant. The full blood count and differential were unchanged except that the lymphocytes appeared to be atypical. The CD4 + was 0.98 (normal range $0.87-1 \cdot 13$ ) and the CD8 + was 1.68 (normal range $0.55-0.69$ ).

The patient was reviewed in the eye clinic during the second week of his penicillin treatment. His visual acuity had improved in each eye to 6/9. Inflammatory cells $(1+)$ were still seen in each anterior chamber. The right vitreous was less cloudy and the left had cleared. Fundoscopy of the right eye showed that the discrete exudates had disappeared, but the retinal oedema persisted. Topical treatment was maintained without the cycloplegic. The patient was seen on two further occasions, at fortnightly intervals, and the eyes continued to improve. His topical treatment was tailed off and, when he was finally seen seven weeks after completion of his ocular and systemic penicillin medication, both eyes were quiet and his visual acuity was $6 / 6$ in each eye.

\section{Discussion}

About $5 \%$ of patients with secondary syphilis develop uveitis, which is bilateral in about half the cases. ${ }^{34}$ Orbital inflammation, conjunctival hyperaemia, optic neuritis, and chorioretinitis may also be present. ${ }^{5}$ The areas of inflammation can be focal or diffuse, or both, and are yellowish-grey in appearance. The peripapillary retina tends to be affected. Retinal vasculitis with retinal haemorrhages may also occur. ${ }^{6}$

In immune compromised patients, retinitis may be caused by infection with herpes simplex, CMV, Toxoplasma gondii, Candida albicans, and possibly HIV.'

In secondary syphilis improvement is prompt after treatment with systemic penicillin and the prognosis for vision is good, although about $10 \%$ of cases have some lasting visual impairment. ${ }^{4}$ Relapses in syphilis have been reported in patients with AIDS. ${ }^{89}$ Our patient's visual and other syphilitic lesions improved within two weeks of commencing penicillin treatment, 
and we therefore consider that his ocular lesions were entirely related to his secondary syphilis. Prompt penicillin treatment is the correct regimen to counter any visual impairment caused by syphilis. In patients with AIDS, however, a longer course of treatment may be necessary. ${ }^{9}$ Our patient has had no further ocular lesions, despite his compromised immune state.

This case report shows the importance of screening for syphilis patients with uveitis and retinitis because, if patients with syphilis were treated with topical or systemic steroids only, the condition could worsen. ${ }^{3}$ It is also of some interest that systemic steroids, taken as a short course, did not apparently accelerate the HIV disease in our patient, as has been reported previously. ${ }^{10}$

We thank Mrs Gaynor Shawcross and Mr Adam Prest of the retinal photographic department, Manchester Royal Eye Hospital, who performed the fundus photography.

\section{References}

1 Passo MS, Rosenbaum JT. Ocular syphilis in patients with human immunodeficiency virus infection. Am $J$ Ophthalmol 1988;106:1-6.

2 Stoumbos VD, Klein ML. Syphilitic retinitis in a patient with acquired immunodeficiency syndrome-related complex. Am $J$ Ophthalmol 1987;103:103-4.

3 MacFaul PA, Catterall RD. Acute choroido-retinitis in secondary syphilis. British Journal of Venereal Diseases 1971;47:159-61.

4 Duke-Elder S. System of ophthalmology. Vol 9. London: Kimpton, 1966:292-321.

5 Spoor TC, Wynn P, Hartel WC, Bryan CS. Ocular syphilis. J Clin Neuro Ophthalmol 1983;3:197-203.

6 Morgan CM, Webb RM, O'Connor GR. Atypical syphilitic chorioretinitis and vasculitis. Retina 1984;4:225-31.

7 Kreiger AE, Holland GN. Ocular involvement in AIDS. Eye 1988;2:496-505.

8 Tramont EC. Syphilis in the AIDS era. $N$ Engl $J$ Med 1988;316:1600-1.

9 Richards BW, Hessburg TJ, Nussbaum JN. Recurrent syphilitic uveitis. N Engl J Med 1989;320:62.

10 Ellis ME, Neal KR, Leen CLS, Newland AC. Alfa-2a recombinant interferon in HIV associated thrombocytopenia. $\mathrm{Br}$ Med $\mathrm{J}$ 1987;295:1519. 